\title{
Interfacial velocities and capillary pressure gradients during Haines jumps
}

\author{
Ryan T. Armstrong* and Steffen Berg \\ Shell Global Solutions International BV, Kesslerpark 1, 2288 GS Rijswijk, The Netherlands
}

(Received 5 June 2013; published 22 October 2013)

\begin{abstract}
Drainage is typically understood as a process where the pore space is invaded by a nonwetting phase pore-bypore, the controlling parameters of which are represented by capillary number and mobility ratio. However, what is less understood and where experimental data are lacking is direct knowledge of the dynamics of pore drainage and the associated intrinsic time scales since the rate dependencies often observed with displacement processes are potentially dependent on these time scales. Herein, we study pore drainage events with a high speed camera in a micromodel system and analyze the dependency of interfacial velocity on bulk flow rate and spatial fluid configurations. We find that pore drainage events are cooperative, meaning that capillary pressure differences which extend over multiple pores directly affect fluid topology and menisci dynamics. Results suggest that not only viscous forces but also capillarity acts in a nonlocal way. Lastly, the existence of a pore morphological parameter where pore drainage transitions from capillary to inertial and/or viscous dominated is discussed followed by a discussion on capillary dispersion and time scale dependencies. We show that the displacement front is disperse when volumetric flow rate is less than the intrinsic time scale for a pore drainage event and becomes sharp when the flow rate is greater than the intrinsic time scale (i.e., overruns the pore drainage event), which clearly shows how pore-scale parameters influence macroscale flow behavior.
\end{abstract}

DOI: 10.1103/PhysRevE.88.043010

PACS number(s): 47.55.nb, 47.56.+r

\section{INTRODUCTION}

The physics of drainage is commonly regarded as well understood and many multiphase flow macroscopic properties are explained by invasion percolation theory [1]. If pore entry pressures and connectivity are known for a given pore morphology then the sequence of pore drainage events, based on local capillary (pore entry) pressure, is deemed predictable. Pore-network simulations take advantage of this predictability by decomposing a porous media into an ensemble of geometric shapes that drain in a sequential process; see e.g., [2-4]. From a thermodynamic perspective, drainage can be further decomposed into an alternation between smooth and reversible displacement followed by spontaneous fluid redistribution [5]. During drainage, potential energy is stored in liquidliquid menisci to a given threshold (i.e., during reversible displacement), at which point energy is released resulting in irreversible fluid rearrangement until a new local energy minimum is obtained (as seen in Fig. 1, a stable meniscus exists at $P_{\text {entry }} 1$ until the applied pressure is greater than $P_{\text {entry }} 1$ at which point energy is released and both Pore 1 and Pore 2 drain). As depicted in Fig. 1, a cascade of pore drainage events is possible if sequentially available pores have entry pressures lower than the initial energy barrier (Pore 1 and Pore 2 drain once $\mathrm{P}_{\text {entry } 1}$ is exceeded). With this geometrical model pore space is artificially subdivided (e.g., Pore 1 and Pore 2 in Fig. 1) which due to accessibility can drain in cascadelike events (Region 1). However, the geometrical model, often used in percolation theory, essentially falls short in the description of pore drainage because the dynamics are not considered and capillarity is assumed to be strictly local (i.e., only the capillary entry pressures of localized individual pores are considered).

Several researchers have studied the drainage process in more detail beyond the percolation picture. Yuan [6] presented

*Ryan.Armstrong@shell.com a unique experimental approach to decomposing pore space into volume fractions relevant to pore drainage. The approach, referred to as apparatus for pore examination (APEX), was a rate-controlled mercury intrusion experiment which divided the pore space into pore bodies (subisons) and pore throats (risons). Subisons are the regions of the capillary pressure curve where pressure increases, after a Haines jump, to the previously obtained capillary pressure $\left(P_{c}\right)$, while risons are the regions of the capillary pressure curve where $P_{c}$ increases to previously unattained values. The volume of fluid associated with a subison corresponds to the volume of void space drained during fluid rearrangement at constant saturation while for a rison this volume of fluid corresponds to the pore neck volume drained prior to the next subison. Using this approach, in combination with x-ray microtomography $(\mu-\mathrm{CT})$, Berg et al. [7] experimentally confirmed that in natural porous rock the volume of fluid associated with a subison is much greater than the volume of a geometrically defined pore body, illustrating that drainage is more appropriately defined by the correlation of entry pressures (i.e., energy barriers) of connected pore regions rather than geometrically constrained pores.

A conceptual model for the dynamics of drainage that considers pore-scale interfacial jumps was presented by Mohanty et al. [8]. The presented model divides a flow

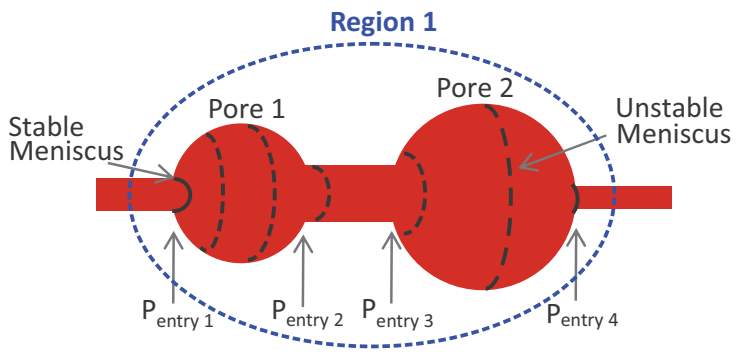

FIG. 1. (Color) A cartoon illustrating pore drainage in a sequence of pores $\left(\mathrm{P}_{\text {entry }} 4>\mathrm{P}_{\text {entry } 1}>\mathrm{P}_{\text {entry } 2}=\mathrm{P}_{\text {entry }} 3\right)$. 
field into various zones. The zone near an advancing front (i.e., the capillary dispersion zone) is called the jump zone while regions further from the front are characterized as a Darcy zone. In the jump zone, fluid properties and local pore geometry determines the time scale of a jump while flow rate determines jump frequency. By taking into account interfacial area (i.e., the fluid-fluid interfacial area along the displacement front) and jump frequency then the average distance between jumps (i.e., along the displacement front) can be obtained and defined as an intrinsic length scale for the zone of influence of simultaneous jumps. Furthermore, Mohanty et al. [8] provides an estimate for jump velocity, in a given pore, by balancing a capillary driving force (i.e., capillary pressure multiplied by pore cross-sectional area) with kinetic energy, which gives a 0.4-ms jump time for a $100-\mu \mathrm{m}$ pore radius. Experimental evidence presented by DiCarlo et al. [9], where acoustic measurements were taken during flow experiments, demonstrate that drainage occurs as discrete fluid bursts originating from pore-scale displacement that occur over the millisecond time scale. Experiments conducted more recently by Moebius and Or [10], utilizing a high speed camera to visualize interfacial jumps during drainage, clearly show behavior not explained by percolation theory. Interfacial oscillations, fluid rearrangement, interfacial pinning, cascadelike pore drainage events, and other interfacial dynamics were found to be dependent on menisci location at the onset of an interfacial jump. Additionally, interfacial velocities during a jump were found to exceed 50 times mean front velocity, thus approaching a flow regime where inertial effects should be considered. These results raise many interesting questions including (a) what controls meniscus dynamics during pore-scale drainage events, (b) to what extent are the dynamics cooperative, (c) how many adjacent pores are affected during an event, (d) how does the dynamics depend on the imposed displacement rate, (e) what are the dominant forces (i.e., inertial, viscous, or capillary) during a drainage event, and (f) what are the macroscale implications?

An often encountered argument, when upscaling from pore scale to continuum scale (via, e.g., a volume average over a representative elementary volume), is that the dynamics of pore-scale events would average out. Some models assume that drainage is overall a percolation process where it is mainly important whether a pore is drained or not and drainage dynamics are not considered. Our experimental observations, presented herein, demonstrate that these often encountered arguments and simplifications are potentially not valid. We find that the interfacial dynamics in relation to the average velocity of the displacement front determines whether a sharp or disperse displacement front develops. Overall, the presented results demonstrate that drainage dynamics at the pore scale influences macroscale behavior and therefore these dynamics should be considered in more detail.

\section{MATERIALS AND METHODS}

The experimental system consisted of a glass micromodel, a syringe pump, an inverted microscope equipped with a high speed camera, and a pair of immiscible fluids. The borosilicate glass micromodel produced by Micronit Microfluidics (Enschede, The Netherlands) was chemically etched with a hexag-
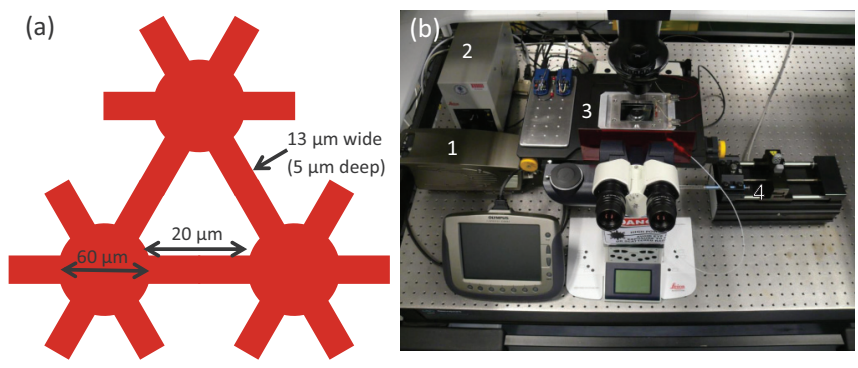

FIG. 2. (Color) Hexagonal micromodel pattern with spherical pore bodies connected to six pore necks (a). Microscope setup (b); high speed camera (1); light source (2); microscope stage with micromodel (3).

onal pore-network pattern [Fig. 2(a)]. Micromodel channels were chemically etched to a depth of $5 \mu \mathrm{m}$, pore bodies were $60 \mu \mathrm{m}$ in diameter, and pore necks were $13 \mu \mathrm{m}$ wide and $20 \mu \mathrm{m}$ in length. The entire flow field was $58 \mathrm{~mm} \times 35 \mathrm{~mm}$, resulting in a total pore volume of $9.5 \mu \mathrm{l}$. The flow rate was controlled with a Harvard Ph.D. Ultra syringe pump allowing for constant flux boundary conditions. Pore drainage events were imaged with an Leica DMI600B inverted transmission microscope equipped with an Olympus iSpeed high speed video camera [Fig. 2(b)]. Images were collected at 2000 frames per second (fps) at $0.5 \mu \mathrm{m} /$ pixel resolution. Additionally, lower resolution images of the entire flow field were collected with an Leica DMI600B inverted fluorescence microscope using a 480/40 $\mathrm{nm}$ bandpass excitation filter, $505 \mathrm{~nm}$ dichromatic mirror, and 527/30 nm bandpass suppression filter. To visualize the entire flow field 120 images $(3840 \times 2880$ pixels $)$ at $1.3 \mu \mathrm{m} /$ pixel resolution were stitched together to create a mosaic.

The wetting phase was Millipore water (with $50 \mathrm{mg} / \mathrm{l}$ fluorescein) and the nonwetting phase was decane (with $35 \mathrm{mg} / 1$ Sudan red). Interfacial tension between the fluid pairs was measured with a DSA 100 Kruss drop shape analysis system. To initiate an experiment, the micromodel was cleaned with $100 \mu \mathrm{l}$ of propanol followed by $100 \mu \mathrm{l}$ of Millipore water. After cleaning, the nonwetting phase was injected and the high speed camera focused on the center of the micromodel was triggered once the invasion front reached the field of view. Flow rates tested were 2, 20, and $200 \mathrm{nl} / \mathrm{min}$, resulting in the following (microscopic) capillary numbers: $3.4 \times 10^{-6}, 3.4 \times 10^{-5}$, and $3.4 \times 10^{-4}$, respectively. When calculating the microscopic capillary number $(\mathrm{Ca}=\mu v / \sigma)$, $\mu$ is the viscosity of the invading phase, $v$ is the average pore velocity of the invading phase (scaled injection flux), and $\sigma$ is the interfacial tension between the immiscible phases.

Images were processed and analyzed with AVIZO 7.1 (Visualization Sciences Group) and TRACKER 4.62 (Open Source Physics) software packages. Interfacial velocities, during a pore drainage event, were measured with the TRACKER software by drawing a reference line across a given pore and tracking the linear distance traveled versus time for a given meniscus. AVIZO was used to create the mosaic images of the entire flow field and to measure interfacial curvature, and thus, capillary pressure (details are presented in $[11,12]$ and references therein). For a micromodel system, only one of the principal radii of curvature of an interface 
$\mathrm{Ca}=3.4 \times 10^{-6}$

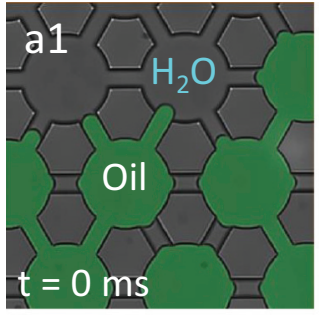

$\mathrm{Ca}=3.4 \times 10^{-5}$

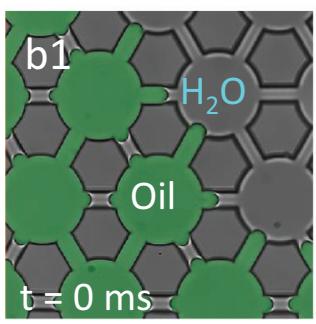

$\mathrm{Ca}=3.4 \times 10^{-4}$

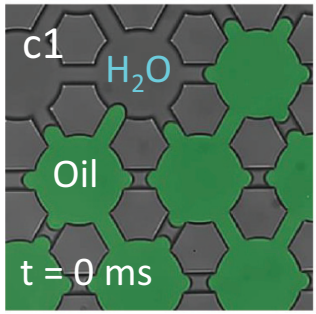

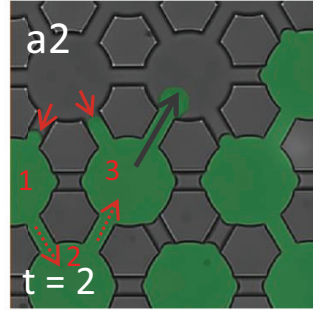
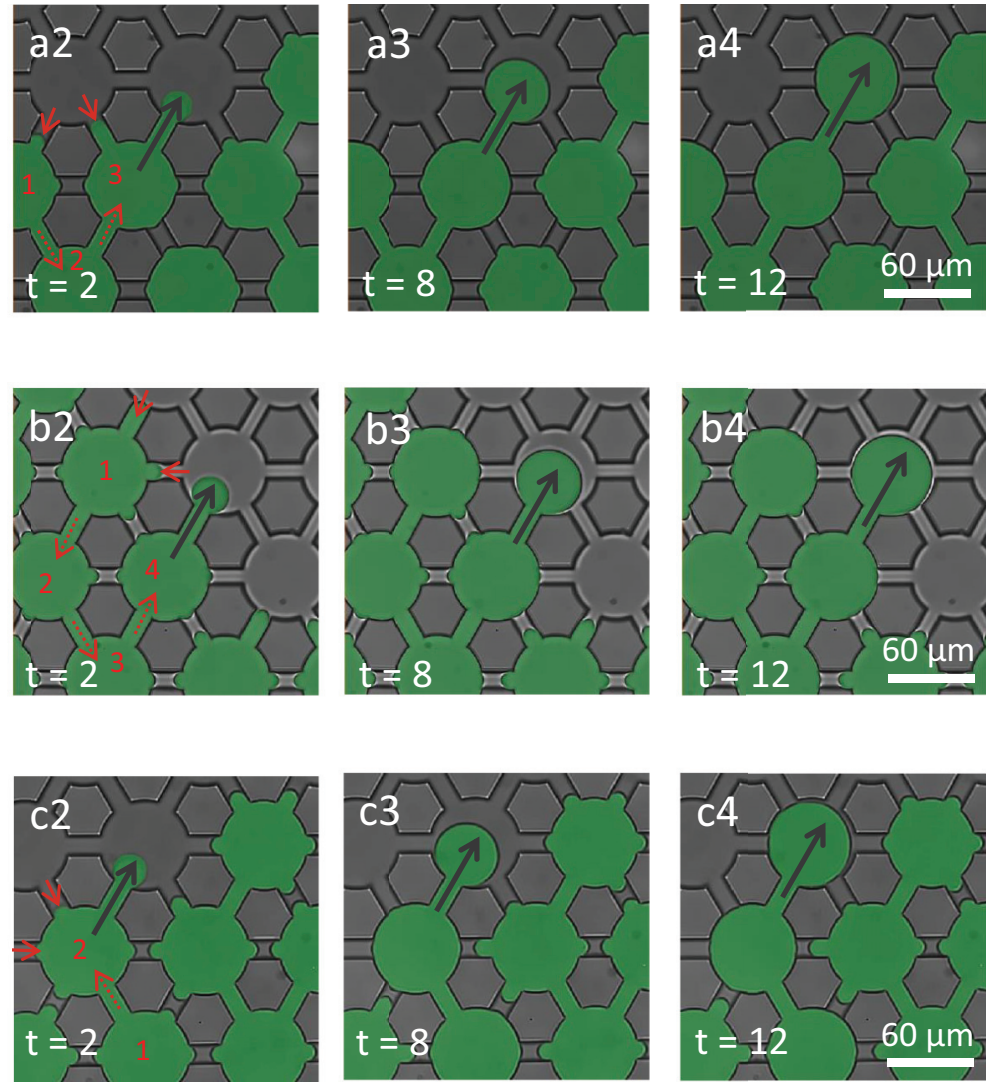

FIG. 3. (Color) High speed images (2000 fps) of pore drainage events at various flow rates (reported in terms of capillary number). Pore drainage events are labeled with black arrows, meniscus retraction is labeled with a red arrow, and the presumed flow direction is labeled with dotted red arrows.

is directly measurable (i.e., the other principal radius of curvature is orthogonal to the focal plan) and thus the second principal radius of curvature must be calculated. To make this calculation, we assume that the contact angle measured for decane-water-borosilicate (using a DSA 100 Kruss drop shape analysis system) is similar to the in situ contact angle in the orthogonal plane during the micromodel experiments. Then capillary pressure $\left(P_{c}\right)$ can be calculated using the Young-Laplace equation stated as

$$
P_{c}=\gamma\left[\left(1 / R_{1}\right)+(2 \cos \theta / d)\right],
$$

where $\gamma$ is interfacial tension, $R_{1}$ is the radius of curvature measured from the collected images, $\theta$ is the measured decanewater-borosilicate contact angle, and $d$ is the micromodel depth.

\section{RESULTS AND DISCUSSION}

Drainage events were imaged with pore-scale resolution at $2000 \mathrm{fps}$ with the high speed camera and selected images, for a range of capillary numbers $\left(3.4 \times 10^{-6}\right.$ to $3.4 \times 10^{-4}$ ), are presented in Fig. 3. By analyzing these images the following observations were made:

(1) A pore drains on the millisecond time scale regardless of the (externally imposed) capillary number.
(2) Pore drainage dynamics are nonlocal (i.e., beyond the draining pore).

(a) During pore body drainage, imbibition occurs in the nearby neck regions.

(b) Menisci retraction is observed over a distance of 4 pores in Figs. 2(b1)-2(b3).

(3) Meniscus curvature in the pore body during a drainage event consistently decreases, which is a function of pore morphology.

(4) Menisci curvatures in the pore neck regions are greater than meniscus curvature in the pore body.

These observations establish that pore drainage events are cooperative and that the individual dynamics, i.e., the time at which a pore is drained, depends on the fluid configuration in adjacent pores beyond the next neighbors. At the capillary numbers tested, drainage events result in liquid rearrangement. As observed, the fluid stored in the nearby pore neck regions supplies a fraction of the fluid necessary for a pore drainage event, which is most evident when comparing images in Figs. 2(a1)-2(a3) [this can also be observed by comparing images (b1) and (b3) or (c1) and (c3)]. These observations are in agreement with the interpretation by Morrow [5] and Yuan [6] pertaining to $\mathrm{Hg}$-air pressure data but here we provide direct visual evidence of the effect. Furthermore, we study these observations in more detail to understand how these cooperative events influence the dynamics of pore drainage. 


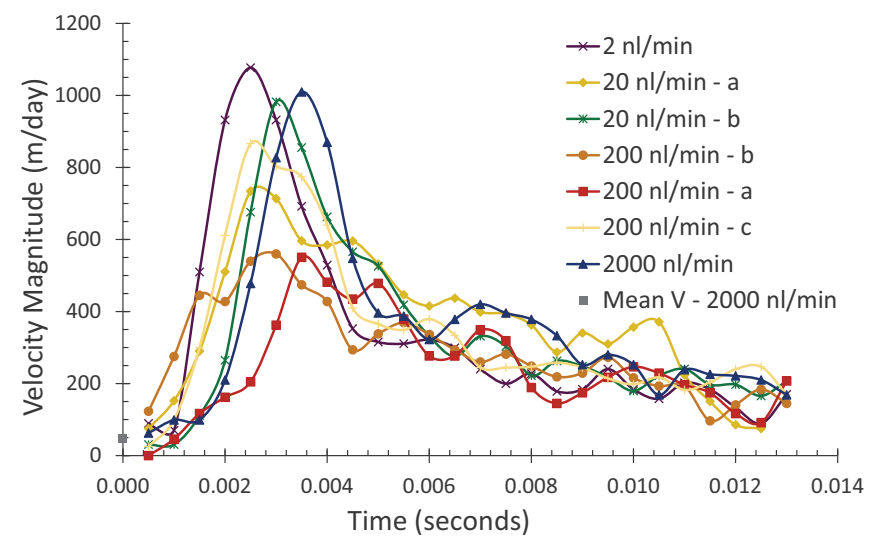

FIG. 4. (Color) Velocity magnitude profile for pore drainage events.

Meniscus velocity was measured by tracking the oil-water interface, across a pore body, during a drainage event. Results presented in Fig. 4, demonstrate that interfacial velocity increases rapidly during the initial one-third time step of an event and then decreases for the duration. The maximum interfacial velocity measured is approximately three orders of magnitude greater than the average mean pore velocity (as calculated from bulk flow rate), which results in a mass flux greater than what can be supplied by the pump. This suggests that pore drainage must be cooperative since the liquid required for a drainage event must be supplied from somewhere other than the external feed. The high interfacial velocity also has implications when modeling drainage at the pore scale since any numerical scheme would require sufficient time resolution to capture these highly dynamic events. Furthermore, even though some drainage events resulted in significantly slower interfacial velocities, there is no correlation between pump flow rate and interfacial velocity (for the range of pump rates tested). For example, essentially the same velocity magnitude profiles are obtained for the 2, 20, and $2000 \mathrm{nl} / \mathrm{min}$ data sets in Fig. 4. This observation is in contrast to results presented by Moebius and Or [10], where event velocity was dependent on bulk flow rate. However, the morphological length scale for our work is $\sim 5 \mu \mathrm{m}$ which (resulting in a capillary-dominated flow regime) is significantly less than the length scale investigated by Moebius and Or [10] which was approximately 1-2 mm. As the length scale of a pore increases the flow regime may shift to a more viscous- or inertial-dominated regime since capillary forces are less dominant and the volume of fluid displaced during an event increases. The consequence is that drainage event velocity becomes dependent on bulk flow rate, as reported by Moebius and Or [10]. Further experiments and/or simulations are required to identify the controlling length scale. However, for sandstone formations, where pore morphological parameters are generally measured in micrometers, it is likely that event velocity is independent of bulk flow rate (i.e., for field relevant displacement velocities) and more dependent on topological properties and surface wettability.

Figure 4 clearly shows that the overall time scale of drainage events is similar but maximum interfacial velocities vary by a factor of 2. To understand the maximum interfacial velocity differences between individual drainage events in pores of almost identical geometry, flow rate, and viscous pressure gradients, the spatial topology of fluid prior to an event was analyzed. Since the pore-scale images, in Fig. 2, suggest that the fluid in the surrounding pore neck regions cooperates with the fluid in the drained pore body it is likely that the volume of fluid available in the nearby neck regions may provide an empirical relationship. Thus, for each pore drainage event the volume of fluid in the adjacent pore neck regions $\left(V_{\text {neck }}\right)$ was measured and then divided by the volume of a pore body $\left(V_{\text {body }}\right)$, which gives the fraction of supplemental fluid readily available for a drainage event [see Fig. 5(a)]. While this approach is phenomenological, the results demonstrate that as the fraction of supplemental fluid increases $\left(V_{\text {neck }} / V_{\text {body }}\right)$ the maximum velocity magnitude of a drainage event increases [see Fig. 5(b)], which gives insight into the relevant physics. The capillary pressure difference, identified in the pore-scale images (see Fig. 2), that occurs during a drainage event should not be overlooked. If sufficient volume is available in the nearby neck regions to supplement the pore drainage event then a steep and local capillary pressure difference (over the length scale of a single pore, i.e., I in Fig. 5) is sustained over a significant fraction of time during the drainage event. However, if the nearby neck regions drain relatively soon after the onset of a drainage event then the local capillary pressure difference will extend over multiple pores (i.e., $>2$ I in Fig. 5) and drainage event velocity decreases. These observations and measurements suggest that the spatial topology of fluids prior to the onset of a drainage event controls the maximum velocity of an event and that neck storage capacity likely dictates rate longevity.

The observed cooperative events and associated fluid topological effects on drainage event velocity raise an interesting fundamental question concerning the locality (i.e., zone of influence) of capillarity during immiscible displacement. The capillary force is traditionally considered to be a local force associated with a single pore. However, differences in capillary forces can result in fluid motion which is referred to as capillarity. The results clearly show that during drainage a capillary pressure difference can exist over multiple pores (shown in Fig. 2 and in more detail in Fig. 7) and this difference and the distance over which it exists can directly affect event dynamics (Fig. 5). As previously shown in Fig. 2, not only the menisci in directly adjacent pores are involved in supplying liquid to the pore drainage event, but that the zone of influence is at least 4 pores from the location where the drainage event occurs. This demonstrates that pore drainage is a rather nonlocal process involving both capillary and viscous forces, over the length scale of multiple pores, which indicates that the approach taken in quasistatic pore-network modeling does not correctly describe the drainage dynamics and associated fluid distributions. Further observations are presented in Fig. 6, where a larger field of view is presented. In Fig. 6(a), multiple menisci contribute to a single pore drainage event and most of the interacting menisci are $>7$ pores from the location of the drainage event. However, the exact distance is not measurable due to field of view and image resolution limitations. In the field of view, many interacting menisci are not connected through the oil phase to the draining pore and are presumably connected beyond the imaged regions. Thus, the total distance over which the capillary force influences fluid topology is not discernible other than stating that this interaction occurs 
(a)

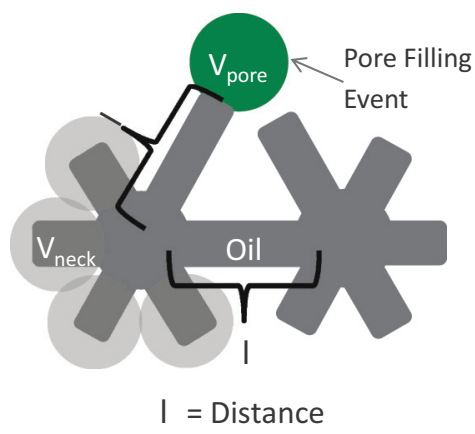

(b)

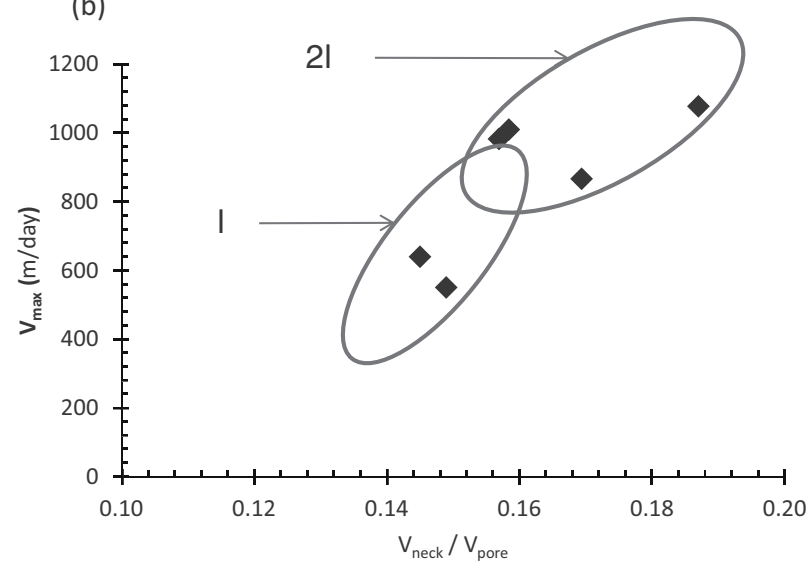

FIG. 5. (Color) Idealized cartoon of pore morphology where $V_{\text {pore }}$ is the volume of a pore body, $V_{\text {neck }}$ is the volume of fluid available in the neck regions of the pore adjacent to a drainage event, and I is the distance between adjacent pores (a). Maximum velocity magnitude measured for a drainage event versus the fraction of supplemental fluid ( $\left.V_{\text {neck }} / V_{\text {pore }}\right)$ readily available for a drainage event (b).

over a distance $>7$ pores. In Fig. 6(b), two pore drainage events occur at essentially the same time [(b1) and (b2)]. As the two menisci advance, one event dominates and inhibits the other event. This observation clearly demonstrates how a pore drainage event can directly influence a meniscus over the distance of multiple pores. However, understanding the zone of influence of a single event is still an open question and is likely dependent on pore morphology and phase connectivity.

To assess the importance of capillarity on interfacial dynamics, we estimate capillary pressure, as a function of time, directly from the images by measuring interfacial curvature [see Eq. (1)]. Pore-scale images collected before, during, and after a meniscus reaches a maximum velocity are displayed in Fig. 7, along with the associated meniscus dynamics and capillary pressure measurements [the images in Fig. 7(a) correspond to the numbered data points in Figs. 7(b) and $7(\mathrm{c})$ ]. It is clearly observed that as the meniscus accelerates the driving capillary pressure $\left(P_{c}\right)$ in the neck region is greater than the opposing $P_{c}$ in the pore body. The drainage event reaches a maximum peak velocity $\left(V_{\max }\right)$ after approximately $1 / 3$ of the pore body is drained, which corresponds to the maximum capillary pressure difference between the measured $P_{c}$ in the draining pore body and the $P_{c}$ in the neighboring pore menisci. Prior to the drainage event, capillary pressure is constant for all menisci and then at the onset of a pore drainage event capillary pressure initially decreases in the

\section{$\mathrm{Ca}=3.4 \times 10^{-5}$}

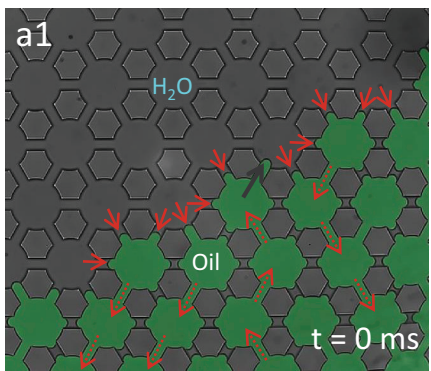

$\mathrm{Ca}=3.4 \times 10^{-3}$

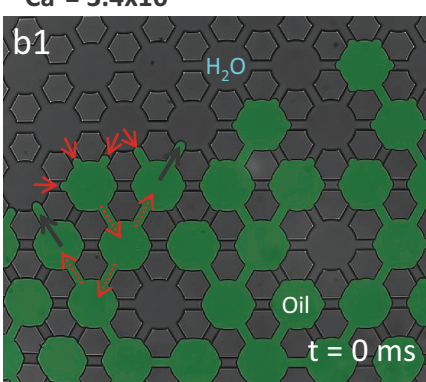

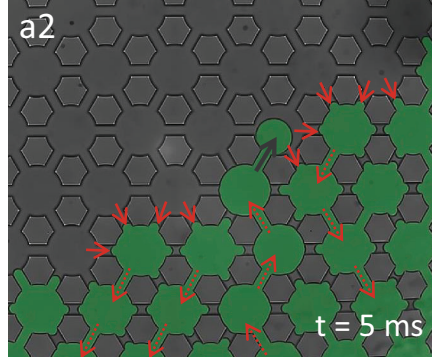
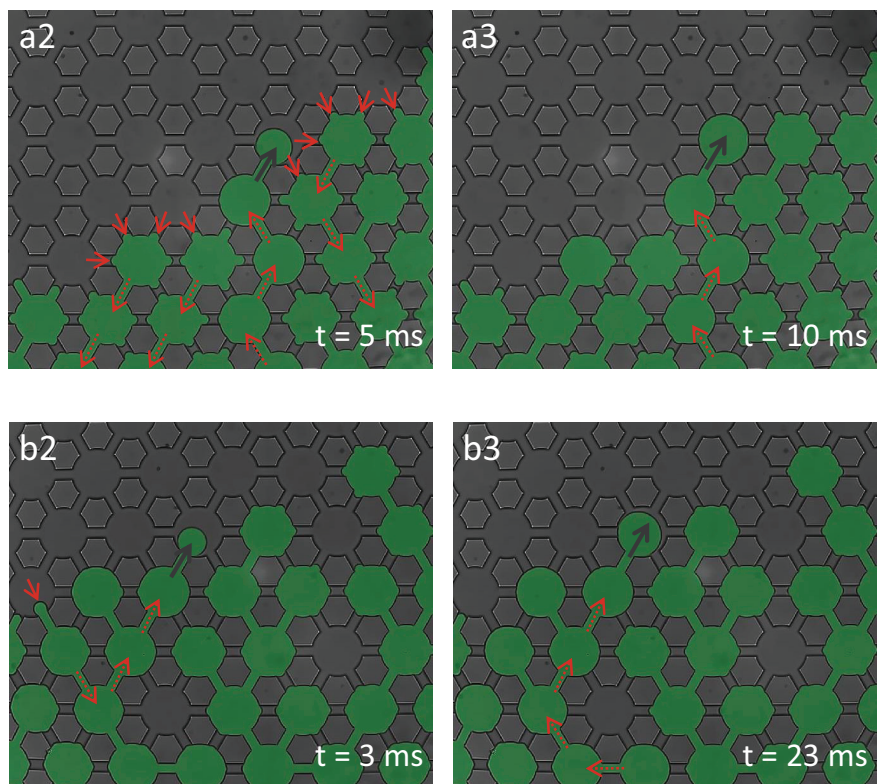

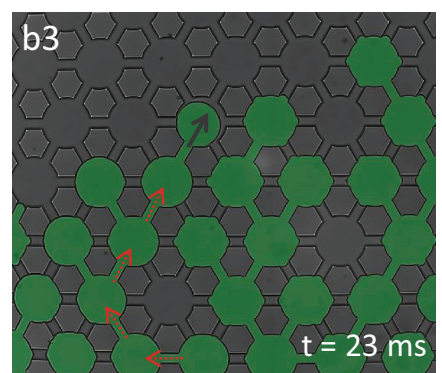

FIG. 6. (Color) Images demonstrating cooperative pore drainage events. Multiple menisci contribute to a single pore drainage event (a). Two pore drainage events are initiated at essentially the same time and then one event dominates and inhibits the other event (b). Pore drainage events are labeled with black arrows, meniscus retraction is labeled with a red arrow, and the presumed flow direction is labeled with dotted red arrows. 
(a)

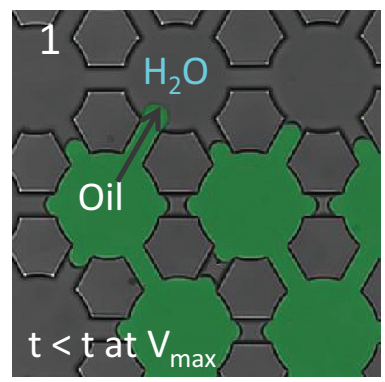

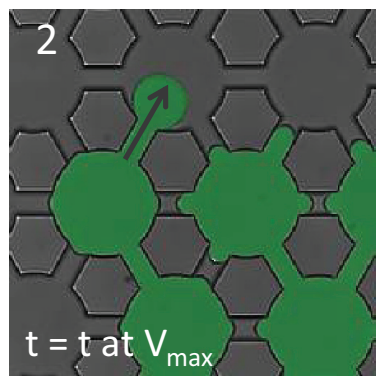

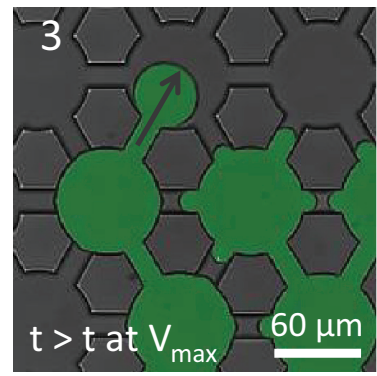

(b)
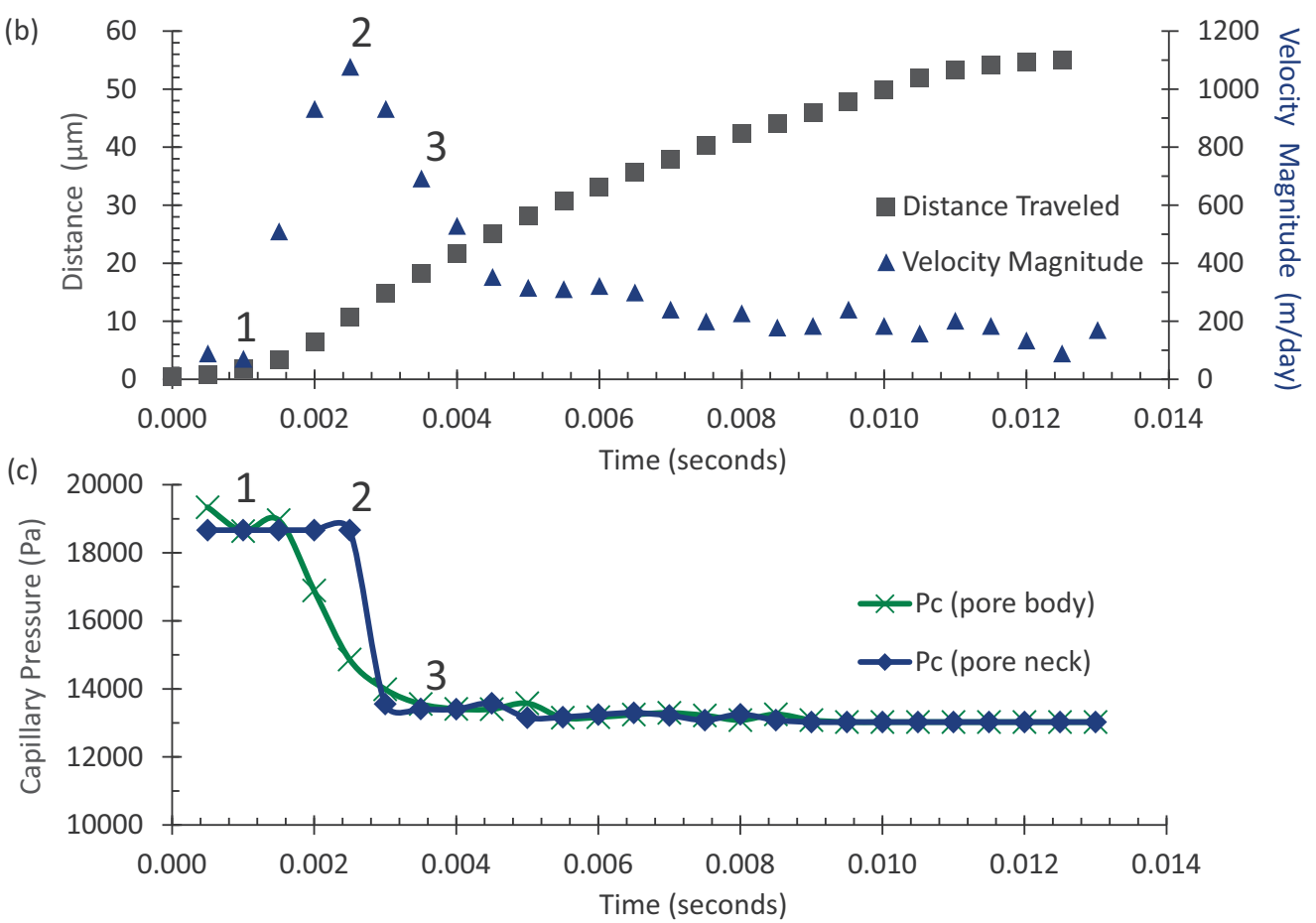

FIG. 7. (Color) Pore-scale images collected before (a1), during (a2), and after (a3) a pore drainage event reaches maximum velocity (a). The distance traveled versus time and meniscus velocity during a pore drainage event (b). The measured capillary pressures in the pore body and in the surrounding menisci during a drainage event (c). The images in (a) correspond to the numbered data points in (b) and (c).

pore body followed by a decrease of capillary pressure in the surrounding pore neck regions [Fig. 7(c)]. At the onset of the pore drainage event, the capillary pressure difference between the pore body meniscus and the surrounding pore neck menisci drives the drainage event forward. While the capillary pressure difference is sustained the meniscus in the pore body accelerates forward until no fluid is available in the neighboring neck regions, at which point the meniscus reaches $V_{\max }$ and the capillary pressure difference in the neighboring region is depleted, and thus a capillary pressure difference from farther-away menisci is required to drive the event to completion. As observed, event dynamics are nonlocal and extend over multiple pores and as fluid is utilized from further pore neck menisci, the distance over which the capillary pressure difference is maintained sequentially increases, which explains the observed deceleration in Fig. 7(b). Therefore, the amount of fluid available in the adjacent pore neck regions will control the time scale over which a large $P_{c}$ difference can be sustained. In more heterogeneous media, it is likely that this cooperative mechanism would lead to significant fluid redistribution.
To parametrize the data, distance traveled by a meniscus (i.e., the meniscus in the draining pore) versus time $(t)$ is presented, as a log-log plot, in Fig. 8(a). From the presented results, it is evident that two distinct regimes exist (i.e., before $V_{\max }$ and after $\left.V_{\max }\right)$. Before $V_{\max }$, the meniscus rapidly advances and distance traveled scales to $\sim t^{3}$. After $V_{\max }$, the meniscus decelerates and distance traveled scales to $\sim t^{1 / 3}$. The same analysis was conducted for all data sets [Figure 8(b)] and mostly consistent results were obtained. Slightly different scaling coefficients were measured for the before $V_{\max }$ regime; for the five data sets with the largest $V_{\max }$ (see Fig. 4) the average power law coefficient was 2.45 (standard deviation $=$ 0.44 ) while the two data sets with the smallest $V_{\max }$ resulted in an average power law coefficient of 1.26 (standard deviation $=$ 0.18 ). For the after $V_{\max }$ regime, similar results were obtained for all data sets with an average power law coefficient of 0.62 (standard deviation $=0.05$ ) regardless of $V_{\max }$. These results support the view that pore drainage is initially dominated by the capillary pressure difference and the length scale over which this difference exists since the power law coefficient for the before $V_{\max }$ regime is rather variable and previously presented 

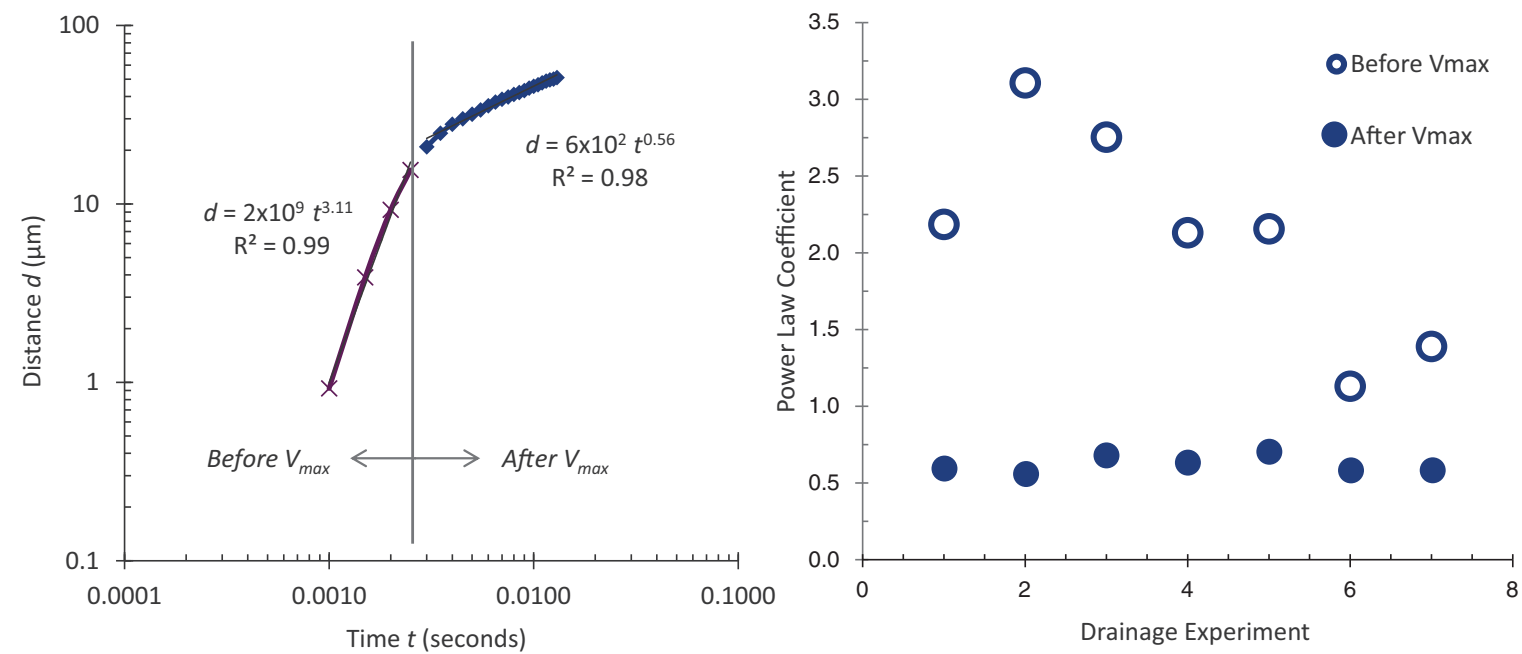

FIG. 8. (Color) Distance traveled versus time for a pore drainage event (a), two distinct power law relationships are evident (i.e., before $V_{\max }$ and after $\left.V_{\max }\right)$. Mostly consistent results were obtained for the measured power law coefficients for distance versus time scaling for all data sets (b).

results demonstrate that this variability is dependent on fluid topology. Whereas, at later times the pore drainage is likely dominated by viscous dissipation and more dependent on fluid properties such as viscosity, since after $V_{\text {max }}$ similar power law coefficients are measured regardless of the magnitude of $V_{\max }$.

Overall, the presented results suggest that at the porescale similar physics are observed for a pore drainage event regardless of bulk flow rate. However, it is well understood, and observed in the presented experiments, that larger-scale displacement patterns are flow rate dependent. To investigate this aspect in more detail, we compared the applied flow rate to the measured pore-scale drainage rate. Considering that pore drainage takes approximately $15 \mathrm{~ms}$ and the total volume of a single pore is $0.014 \mathrm{nl}$, then a single drainage event requires a mass flow rate of $56 \mathrm{nl} / \mathrm{min}$. Therefore, in the presented experiments, the applied flow rates are both above $(200 \mathrm{nl} / \mathrm{min})$

(a)
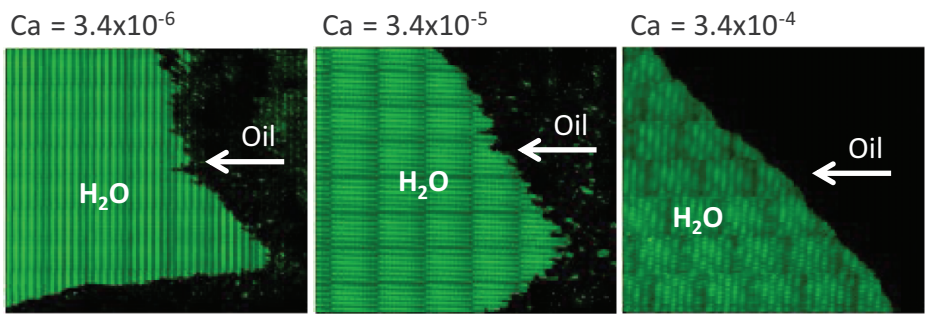

Intrinsic rate
$R_{i}=\frac{\text { pore volume }}{\text { drainage time }}$
$R_{i}=56 \mathrm{nl} / \mathrm{min}$

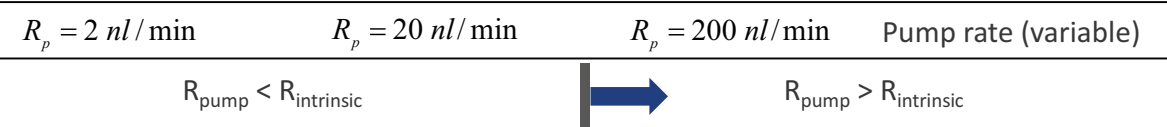

(b)

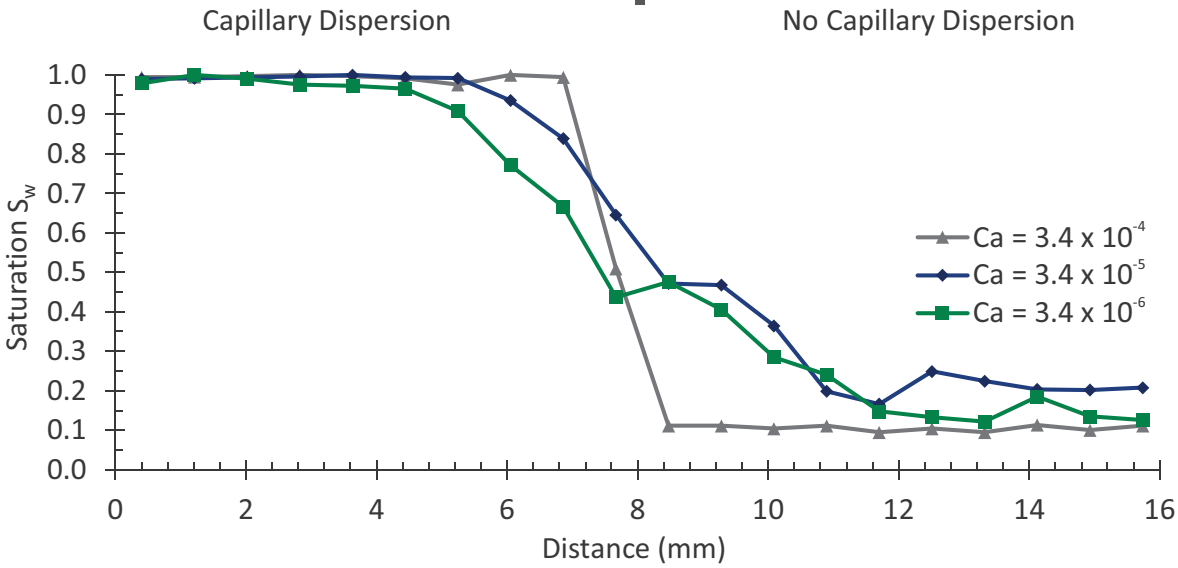

FIG. 9. (Color) Displacement stability for the micromodel experiments (a). Saturation profiles (calculation is based on average saturation for a 16-mm zone over the main displacement region) for the micromodel experiments (b). 
and below ( 2 and $20 \mathrm{nl} / \mathrm{min}$ ) the intrinsic rate required for a drainage event. In Fig. 9, mosaic images of the flow field, taken during micromodel drainage, at applied flow rates less than and greater than the intrinsic rate required for pore drainage are presented. The images [Fig. 9(a)] and corresponding saturation profiles [(Fig. 9(b)] illustrate that at applied flow rates less than the intrinsic rate capillary dispersion occurs. However, as the applied rate becomes greater than the intrinsic rate capillary dispersion is inhibited (i.e., the applied rate dominates). This observation highlights the importance of competing time scales and describes a correlation between pore-scale events and larger-scale rate dependencies. We hypothesize that by pumping at a rate greater than the intrinsic rate either the system is not allowed time to reach capillary equilibrium or that the intrinsic length scale for the zone of influence of simultaneous jumps, as proposed by Mohanty et al. [8], decreases such that interference between individual events occurs which suppresses capillary dispersion and thus results in a steeper front. However, further work is required for validation and development of this concept. Additionally, it should be noted that for heterogeneous porous media a distribution of intrinsic rates would apply and thus a single transition point where capillary dispersion is suppressed may not exist.

From what is known in the literature, the controlling parameters for displacement front behavior are mobility ratio and capillary number. For the presented experiments, the mobility ratio is $\sim 1(\log M=0)$ and the capillary number ranges from $-6<\log \mathrm{Ca}<-4$. When comparing these values to the Lenormand phase diagram from Zhang et al. [13], as capillary number increases, the presented experiments would transition from the capillary fingering regime to the neutral territory between the capillary fingering and the stable regime. Thus, capillary number and mobility ratio can explain the flow regime transition observed in Fig. 9. In the end, both pictures (i.e., phase diagram and intrinsic time scales) are actually compatible since the externally applied flow rate controls the viscous force and the intrinsic time scale is capillary controlled. However, the presented time scale perspective provides a means to characterize the capillary force for different pore morphologies, and thus is potentially extendable, whereas the Lenormand phase diagram is unique to a given pore morphology.

\section{CONCLUSIONS}

The dynamics of pore drainage events were studied in a two-dimensional micromodel at low and intermediate capillary numbers using a high speed camera which explicitly resolves meniscus propagation. It was found that pores with a diameter of $60 \mu \mathrm{m}$ were drained typically in $15 \mathrm{~ms}$. Drainage dynamics were found to be cooperative, i.e., involving adjacent pores in the sense that menisci in adjacent pores would retreat during an event and the speed of meniscus propagation was dependent on the amount of nonwetting fluid "stored" in liquid-liquid menisci in the vicinity of an event. Concerning meniscus propagation, we observe two distinct regimes for meniscus position versus time: (1) before $V_{\max }$ and (2) after $V_{\max }$. These two regimes are independent of bulk flow rate and are rather likely a function of pore morphology (e.g., neck storage capacity, aspect ratio, and/or surface wettability) and potentially fluid properties (e.g., viscosity, density, and interfacial tension).

However, whereas the observed pore-scale processes were independent of bulk flow rate we observe larger-scale patterns which are flow rate dependent. Concerning capillary dispersion of the macroscopic fluid front we observe that when pumping at a flow rate greater than the intrinsic rate required for pore drainage the front becomes sharp compared to a disperse front at lower pumping rates. It is likely that many of the rate dependencies observed in multiphase flow are indeed caused by competing time scales (i.e., the time scale for a drainage event and the time scale for bulk flow) where an applied rate controls which processes are allowed to approach equilibrium.

From the presented experiments the following conclusions are possible:

(1) The velocity of a pore drainage event is controlled by its intrinsic dynamics, defined by capillary-viscous-inertial processes and independent of the bulk flow rate. The internal time scale is defined by the local geometry and fluid distribution and topology in adjacent pores.

(2) A capillary pressure difference drives the pore drainage event forward and the spatial arrangement of fluid controls the rate at which pores are drained.

(3) From the onset of a drainage event, the event is driven to completion by capillary forces in the pore neck regions.

(a) The capillary force difference extends over multiple pores which makes capillarity effectively a nonlocal phenomenon.

(b) Nonwetting fluid in pore neck menisci is utilized during a drainage event and the radius of influence is $>7$ pores.

(4) Distance traveled versus time for a meniscus during a drainage event has two distinct regimes. However, in more natural porous media this might be less distinctly separated.

(5) Overall, capillary dispersion was dependent on the ratio of the applied and intrinsic rate for pore drainage.

(6) The presented time scale perspective, which does not contradict the conventional capillary versus viscous force balance view, provides a means to characterize the capillary force for different pore morphologies, and thus is potentially extendable, whereas the Lenormand phase diagram is unique to a given morphology.

(7) Consequently, pore-scale dynamics potentially influences macroscale behavior.

These results set the foundation for further work on understanding the time scales relevant to pore-scale drainage events.

From the presented experiments the following open questions are proposed:

(I) Is there a pore morphological length scale at which flow transitions to a regime where inertial forces are important, such that drainage event velocity becomes dependent on bulk flow rate?

(II) Can pore drainage events be overrun by bulk flow rate when the applied rate is significantly larger (i.e., greater than the flow rates presented in this work)?

(III) What is the radius of influence of the capillary pressure difference during a pore drainage event? 
Likewise, at what distance does a drainage event cause menisci movement and what implications does this have when defining a two-phase representative elementary volume?

(IV) How does the cooperative nature of a pore drainage event depend on pore morphology (in particular, pore connectivity and pore body to throat aspect ratio)?

(V) Can the energy remaining from a single pore drainage event initiate sequential events in adjacent pores or is the total energy generated dissipated during a single event (i.e., all fluids come to a rest)?

(VI) In a real porous system, what is the distribution of intrinsic pore drainage rates? How well do they coincide with rates based on pore geometry?

(VII) Does the observed correlation between capillary dispersion and the ratio between pumping rate and intrinsic pore drainage rate hold when there is a distribution of intrinsic rates or is this a special effect only observed in highly regular and monodisperse systems?

\section{ACKNOWLEDGMENTS}

Katie Humphry is gratefully acknowledged for the design of the micromodel pattern. Rob Neiteler is acknowledged for the design of the micromodel holder. Additionally, we would like to acknowledge Open Source Physics which is supported in part by the National Science Foundation Grants No. DUE0126439 and No. DUE-0442481 and Douglas Brown for developing the Tracker Video Analysis and Modeling Tool. Lastly, we thank Vahid Joekar-Niasar, Cor van Kruijsdijk, and Apostolos Georgiadis for valuable discussions regarding capillarity.
[1] B. Berkowitz and R. Ewing, Surv. Geophys. 19, 23 (1998).

[2] I. Fatt, Petroleum Trans. AIME 207, 144 (1956).

[3] D. Wilkinson and J. Willemsen, J. Phys. A 16, 3365 (1983).

[4] J. Vogel, Eur. J. Soil Sci. 51, 99 (2000).

[5] N. Morrow, Ind. Eng. Chem. 62, 32 (1970).

[6] H. H. Yuan, SCA Fourth Annual Technical Conference, August 15-16, 1990 (Society of Core Analyst, 1990), Paper No. 9004.

[7] S. Berg, H. Ott, S. A. Klapp, A. Schwing, R. Neiteler, N. Brussee, A. Makurat, L. Leu, F. Enzmann, J. O. Schwarz, M. Kersten, S. Irvine, and M. Stampanoni, Proc. Natl. Acad. Sci. USA 110, 3755 (2013).
[8] K. K. Mohanty, H. T. Davis, and L. E. Scriven, SPE Reservoir Eng. 9406, 113 (1987).

[9] D. A. DiCarlo, J. I. G. Cidoncha, and C. Hickey, Geophys. Res. Lett. 30, 17 (2003).

[10] F. Moebius and D. Or, J. Colloid Interface Sci. 377,406 (2012).

[11] R. T. Armstrong, M. L. Porter, and D. Wildenschild, Adv. Water Resour. 46, 55 (2012).

[12] R. T. Armstrong, C. Pentland, S. Berg, J. Hummel, D. Lichau, and L. Bernard, in Proceedings of International Symposium of the Society of Core Analysts, Aberdeen, Scotland, UK, 27-30 August, 2012 (Society of Core Analyst, 1990).

[13] C. Zhang, M. Oostrom, T. W. Wietsma, J. W. Grate, and M. G. Warner, Energy Fuels 25, 3493 (2011). 